\title{
Variación espacio-temporal de Scleractinia, Gorgonacea, Gastropoda, Bivalvia, Cephalopoda, Asteroidea, Echinoidea y Holothuroidea, de fondos someros de la isla San José, Golfo de California
}

\author{
Oscar Efraín Holguin Quiñones ${ }^{1}$, Felipe de Jesús González Medina ${ }^{1}$, Francisco Solís Marín ${ }^{2}$ \& \\ Esteban F. Félix Pico ${ }^{1}$ \\ 1. Centro Interdisciplinario de Ciencias Marinas, Instituto Politécnico Nacional, Apdo. Postal 592. La Paz, B.C.S., \\ México; oholguin@ipn.mx, glezmedina@yahoo.com,efelix@ipn.mx \\ 2. Laboratorio de Sistematica y Ecologia de Equinodermos. Instituto de Ciencias del Mar y Limnologia. Universidad \\ Nacional Autónoma de Mexico. Apdo. Post. 70-305, México, D.F. 04510, México; fasolis@icmyl.unam.mx
}

Recibido 03-VII-2007. Corregido 30-VI-2008. Aceptado 31-VII-2008.

\begin{abstract}
Space-time variation of Scleractinia, Gorgonacea, Gastropoda, Bivalvia, Cephalopoda, Asteroidea, Echinoidea and Holothuroidea, from shallow bottom in San Jose Island, Gulf of California. Between the years 1999 and 2000, six expeditions were made to Isla San José, in the Gulf of California. Observations and samples were taken of benthic macroinvertebrates in nine locations. Based on the samples, the specific components of three phyla were determined: Cnidaria (Scleractinia and Gorgonacea), Mollusca (Gastropoda, Bivalvia and Cephalopoda), and Echinodermata (Echinoidea and Holothuroidea). The abundance of conspicuous benthic macroinvertebrates was visually estimated through free dives at a depth of between two and six meters along two transects parallel to the coast. A total of 38 taxa in three groups were determined. The most abundant species are Tripneustes depressus (44,9\%), Echinometra vanbruntii (18,3\%), Phataria unifascialis $(8,9 \%)$, Centrostephanus coronatus $(8,0 \%)$, Diadema mexicanum (5\%) y Eucidaris thouarsii (3,6\%). No significant differences between diversity $\left(\mathrm{H}^{\prime}\right)$, equity $(\mathrm{J})$ and richness of the species $(\mathrm{S})$ during the months and site of study. The similarity analysis identified two groups from locations of the eastern and western coasts. Rev. Biol. Trop. 56 (3): 1189-1199. Epub 2008 September 30.
\end{abstract}

Key words: Macroinvertebrates, diversity, San José Island, Gulf of California.

La costa oriental del estado de Baja California Sur e islas cercanas tienen elevada diversidad biológica y abundancia de recursos bentónicos, como lo demuestran los estudios realizados en los últimos veinte años, especialmente en las bahías de La Paz y áreas circunvecinas por investigadores del CICIMAR y otras instituciones de la región (Schwartzlose et al. 1992, González-Navarro y Elorduy 1997, Maluf y Brusca 2005, Hendrickx y Brusca 2005).

Entre los invertebrados marinos, los moluscos, equinodermos y corales son grupos muy conocidos en el golfo de California. La isla San José, al igual que otras grandes islas cercanas a la ciudad de La Paz (Espíritu Santo y Cerralvo) es objeto de explotación permanente de sus recursos marinos, de manera tal que en diversas especies de interés económico, principalmente moluscos, se observan altos grados de sobreexplotación (Holguin-Quiñones y García-Domínguez 1997).

Las aguas circundantes a este cuerpo insular, sabemos ahora, albergan diversidad de especies aprovechables y potencialmente explotables. No obstante, esta isla ha sido muy poco investigada y se desconocen muchos aspectos relacionados con la composición y estructura de las comunidades de invertebrados de fondos rocosos someros que nos permitan emitir juicios fundados acerca de su potencial biológico.

El objetivo de este trabajo es aportar información biológico-ecológica de fauna de invertebrados en fondos someros rocosos de la isla San José. 
Algunos autores mexicanos han llevado a cabo estudios puntuales en la costa oriental e islas vecinas a este litoral de Baja California Sur (Baqueiro y Massó 1988, Baqueiro et al. 1981, Cintra-Buenrostro et al.1999, GarcíaDomínguez 1991, García-Domínguez y FélixPico 1993, González-Medina et al. 2006, Holguin-Quiñones et al. 2000, 2002, LandaJaime et al. 2005, Reyes-Bonilla y López Pérez 1998, Reyes-Bonilla et al. 2005, Solís-Marín et al. 1993, 1997 y 2005, entre otros). No obstante, todavía hoy, una de las islas menos estudiadas y conocidas es precisamente San José sobre la cual no se cuenta con información publicada referente a aspectos biológico-ecológicos y de pesquerías de invertebrados a pesar de que está fisiográficamente muy relacionada con la bahía de La Paz.

Los corales, moluscos y equinodermos son grupos muy conocidos en el golfo de California en donde se han registrado alrededor de 2680 especies actuales (Morris 1966, Maluf 1988, Keen 1971, Brusca 1980, Maluf y Brusca 2005). Entre los moluscos se han reconocido 1526 especies de la Clase Gastropoda, 553 de la Clase Bivalvia y 20 de la Clase Cephalopoda ( Keen 1971, Brusca 1980, Hendrickx y Brusca 2005), de equinodermos se conocen 63 especies de la Clase Asteroidea, 47 de Echinoidea y 57 de Holothuroidea (Holguin-Quiñones et al. 2000, Maluf 1988, Maluf y Brusca 2005,
Solís-Marín et al. 1993, 1997, Solís-Marín y Laguarda 2005), y de corales pertenecientes a la Clase Anthozoa 39 especies del Orden Scleractinia (corales duros) y 13 del Orden Gorgonacea (Durham y Barnard 1952, ReyesBonilla et al. 2005, Brusca y Trautwein 2005).

\section{MATERIALES Y MÉTODOS}

La isla San José (Fig. 1) se encuentra en los $24^{\circ} 59^{\prime}$ latitud norte y $110^{\circ} 37^{\prime}$ longitud oeste, está ubicada en el golfo de California, al norte de la bahía de La Paz, separada del macizo peninsular por el estrecho canal de San José, mide $28 \mathrm{~km}$ de largo por 7,5 km de ancho y es de las islas más grandes de la costa oriental de Baja California Sur. Su costa presenta acantilados en la parte occidental, algunas caletas protegidas y pequeñas playas. La costa en sus porciones oriental y sur tiene playas arenosas extensas.

Se realizaron seis expediciones a la isla entre los años 1999 y 2000 con periodicidad bimensual; se trabajó en 9 puntos de muestreo permanentes (Fig. 1) en la franja costera. En cada localidad se llevó a cabo una prospección visual y recolección de especímenes de los tres grupos bajo estudio en fondos someros, se estimaron densidad y abundancia de equinodermos, moluscos y corales y se cuantificaron las especies más conspicuas mediante el sistema

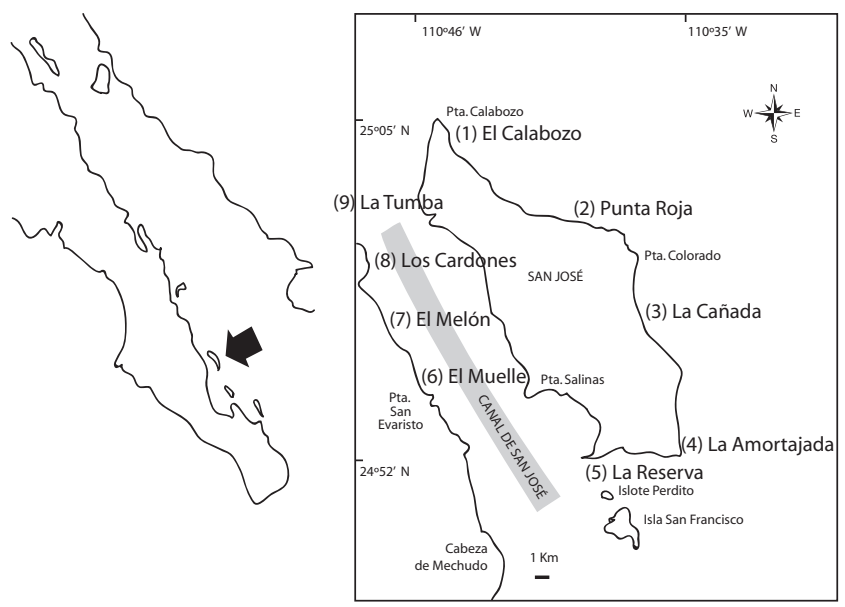

Fig. 1. Mapa del área de estudio y ubicación de las localidades de muestreo en la Isla San José, México. 
de transectos en banda (Conquest et al. 1996), a profundidades entre dos y seis metros (50 $\mathrm{m}$ x $5 \mathrm{~m}$ con repetición) perpendiculares a la costa en cada uno de los puntos de muestreo. Las observaciones se realizaron por medio de buceo libre. El número de individuos avistados por especie (epifauna) se anotó en tablillas de acrílico para cada área de observación.

Con este sistema se hizo un conteo de los especímenes a lo largo del transecto en un campo visual de 2,5 $\mathrm{m}$ a derecha e izquierda, lo cual representó un área de $500 \mathrm{~m}^{2}$ por punto de muestreo y fecha de observación. El área total de evaluación durante cada expedición a la isla fue de $4500 \mathrm{~m}^{2}$.

Se coleccionaron algunos especímenes de dudosa identificación. Fueron fijados con solución de formaldehído al $10 \%$ con excepción de los holoturoideos, los cuales se fijaron en alcohol al 70\%. Para la determinación de las especies se utilizaron los trabajos de Caso (1979, 1994), Brusca (1980), Gotshall (1987) y Kerstitch y Bertsch (2007). Los especimenes fueron depositados en la colección de referencia de invertebrados del CICIMAR-IPN.

Para efectuar el análisis estadístico de la estructura comunitaria se utilizó solo la abundancia de equinodermos y moluscos. Debido a que los corales están compuestos por colonias fueron evaluados en porcentaje de cobertura. Se emplearon métodos univariados y multivariados para hacer comparaciones entre las localidades y meses estudiados. La riqueza de especies $(S)$ fue representada mediante el número total de las especies en una muestra, la diversidad se obtuvo mediante el índice de Shannon-Weaver $\left(H^{\prime}\right)$ empleando el Log 2 (Magurran 1988), la equidad $\left(J^{\prime}\right)$ con el índice de Pielou (1975). La matriz de similitud de Bray-Curtis (1957) de las localidades se construyó transformando los datos de abundancia por especie con raíz cuarta ( $\sqrt[4]{ }$ ) (Field et al. 1982), mientras que la clasificación se realizó empleando la estrategia de unión promedio simple mediante el programa BioDiversity Professional Beta (McAleece 1997). Se aplicaron análisis de variancia (ANOVA; $\mathrm{NC}=95 \%$ ) de dos vías a la diversidad, equidad y riqueza para determinar si existían diferencias significativas entre las localidades y muestreo, previa comprobación de la normalidad y homogeneidad de varianzas. Dichos análisis se realizaron mediante el programa STATISTICA (StatSoft Inc. 2003).

\section{RESULTADOS}

La composición específica de fondos someros de las localidades muestreadas en la isla San José dentro y fuera de transecto comprende 38 especies agrupadas en tres grandes grupos: Mollusca, 16 táxones (42,1\%), Echinodermata, 15 táxones $(39,5 \%)$ y Cnidaria, 7 táxones $(18,4 \%)$.

Los indicadores estructurales de la comunidad $\left(\mathrm{H}^{\prime}\right.$ diversidad, $\mathrm{J}^{\prime}$ equidad y $\mathrm{S}$ riqueza de especies) reflejan muy poca fluctuación espacial y temporal como se muestran en las figuras 2 A y B. En general, se observa poca variabilidad a lo largo del periodo de muestreo en estos tres índices, no existiendo diferencias significativas entre los meses para la diversidad $\left(H^{\prime}\right)$ (ANOVA, $\mathrm{F}=1.7219, \mathrm{p}=0.1892$ ), equidad $(J)(\mathrm{F}=1.9367, \mathrm{p}=0.1506)$ y riqueza de especies $(S)(\mathrm{F}=0.2470, \mathrm{p}=0.8627)$ y para las localidades de muestreo tampoco se encontraron diferencias significativas para la diversidad $(\mathrm{F}=1.2815, \mathrm{p}=0.2989)$, equidad $(\mathrm{F}=1.5626$, $\mathrm{p}=0.1886)$ y riqueza de especies $(\mathrm{F}=1.8671$, $\mathrm{p}=0.1132)$.

Con respecto a la abundancia relativa promedio por localidades, las especies más abundantes son Tripneustes depressus (40,5\%), Echinometra vanbruntii (21,3\%), Phataria unifascialis (8,0\%), Centrostephanus coronatus (7,2\%), Diadema mexicanum (4,5\%) y Eucidaris thouarsii $(3,7 \%)$, las cuales representan un $85 \%$ de la abundancia de la comunidad (Fig. 3).

Mediante el análisis de similitud entre las estaciones de muestreo es posible ver en el dendrograma a un nivel de corte del $75 \%$, una clara separación entre las localidades del litoral occidental menos expuesto al oleaje ( $\mathrm{La}$ Tumba, Los Cardones, El Muelle y El Melón) debido a que se encuentran ubicadas frente 

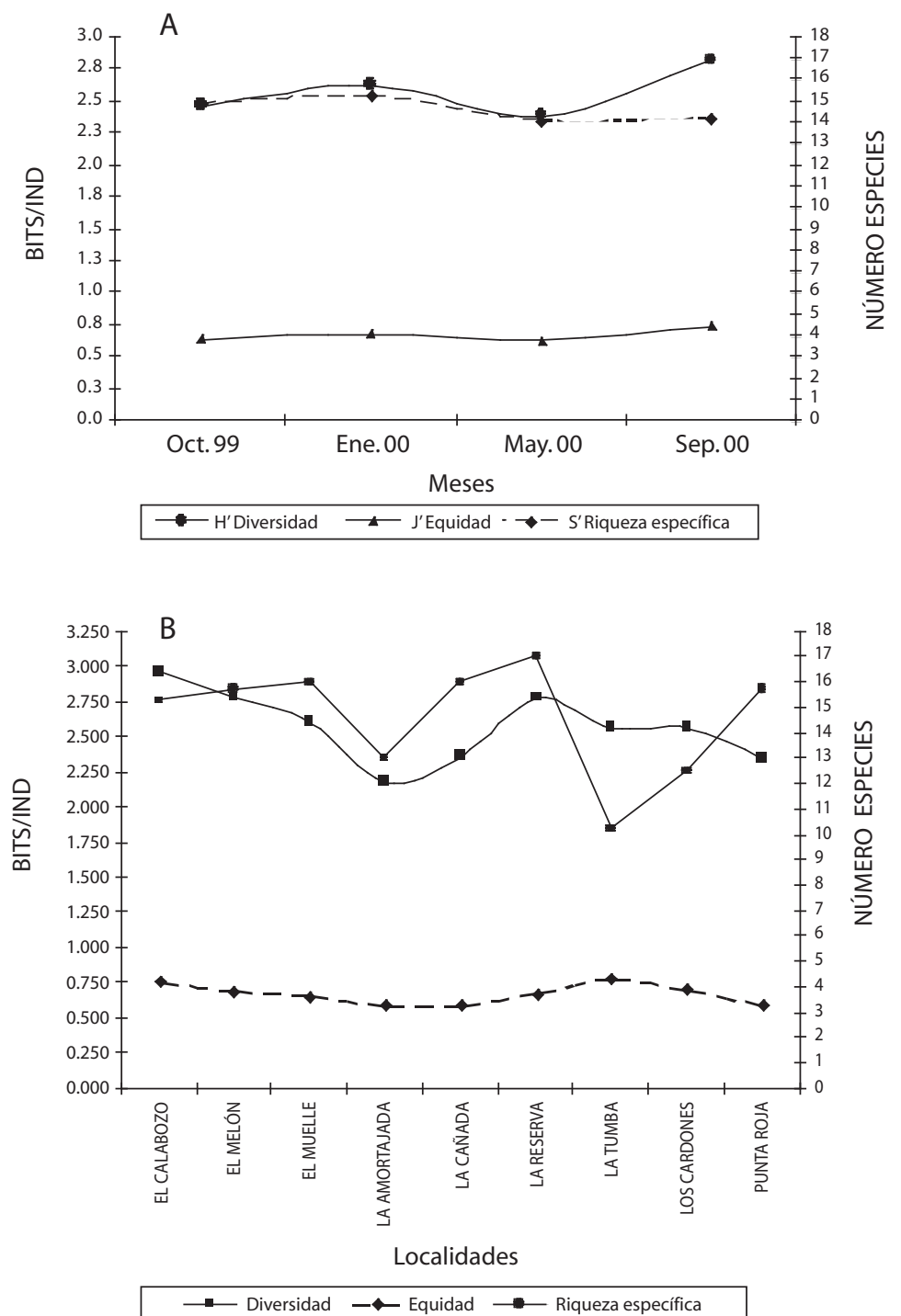

Fig. 2. A Fluctuaciones en la diversidad $\left(H^{\prime}\right)$, equidad $(J)$ y riqueza de especies $(S)$ entre los cuatro meses de muestreo. B. Fluctuaciones en la diversidad $\left(H^{\prime}\right)$, equidad $(J)$ y riqueza de especies $(S)$ entre las nueve localidades de muestreo.

al canal de San José y las del litoral oriental que por estar ubicadas hacia mar abierto tienen mayor nivel de exposición al oleaje ( $\mathrm{La}$ Reserva, La Cañada, La Amortajada, Punta Roja y El Calabozo) (Fig. 4).

\section{DISCUSIÓN}

De las 15 especies de equinoideos registradas en este trabajo para la isla San José, la más abundante (Tripneustes depressus, abundancia relativa 40,5\%) corresponde al grupo de especies características del golfo de California mencionada por Solís-Marín et al. (2005), el mismo caso lo presenta el asteroideo Phataria unifascialis (abundancia relativa 8,0\%), que también es una especie característica del golfo de California, junto con Pentaceraster cuminigi y Pharia pyramidatus. En cuanto a las dos especies de holoturoideos presentes en 


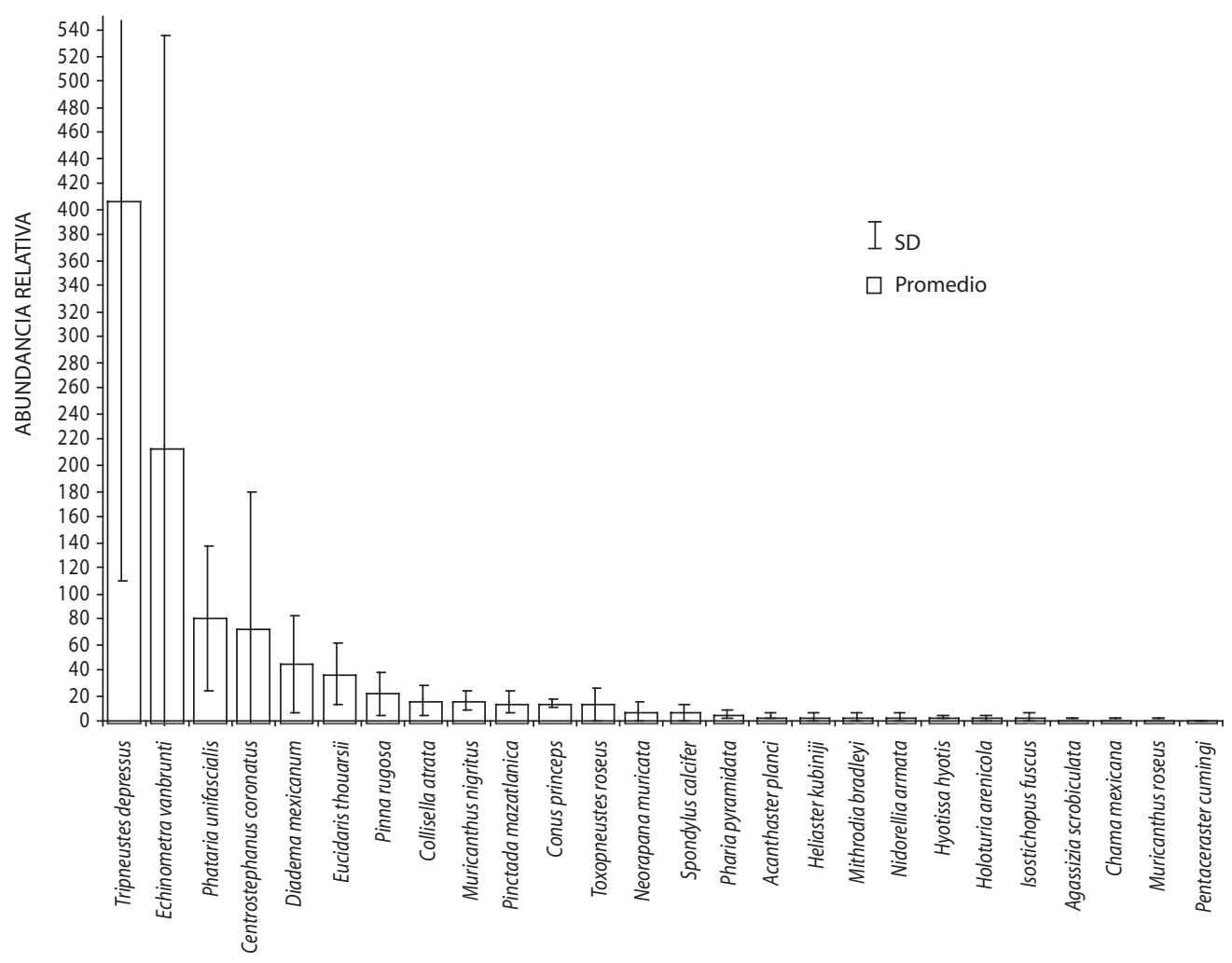

Especies

Fig. 3. Valores promedio (barra) y desviación estándar (líneas) de la abundancia media (individuos) de las especies de macroinvertebrados de la Isla San José, México.

el estudio, el "pepino arenero" Holothuria (Thymiosycia) arenicola presentó valores bajos de abundancia relativa en relación al resto de los equinodermos. Esta especie se halla distribuida, tanto en las costas orientales del golfo de California (Sonora y Sinaloa), como en el golfo de México y en el caribe Mexicano.

Es importante subrayar que no se observaron ofiuroideos ya que estos organismos son de hábitos crípticos y de fototactismo negativo, lo cual los hace esconderse debajo de piedras, y durante la realización de los transectos en banda no se removió, en ningún caso, el substrato.

La riqueza de especies de equinodermos registrada en los fondos rocosos de la isla
San José, resultó, como debería de esperarse, menor a lo encontrado por distintos autores en ambientes de arrecifes coralinos: en el arrecife de Cabo Pulmo, B.C.S. se han registrado 38 especies (Cintra-Buenrostro et al. 1999); para las zonas arrecifales de Tenacatita, Jalisco se han registrado 17 especies (Landa-Jaime et al. 2005), y para la zona de La Entrega, Oaxaca, se han reportado 20 especies (Zamorano y LeyteMorales 2005). La composición específica de equinodermos de la zona tiene gran afinidad, aunque menor número de especies, a lo reportado por Holguin-Quiñones et al. (2000) para la Bahía de Loreto (26 especies).

La fauna de equinodermos del golfo de California conocida hasta la fecha, presenta 


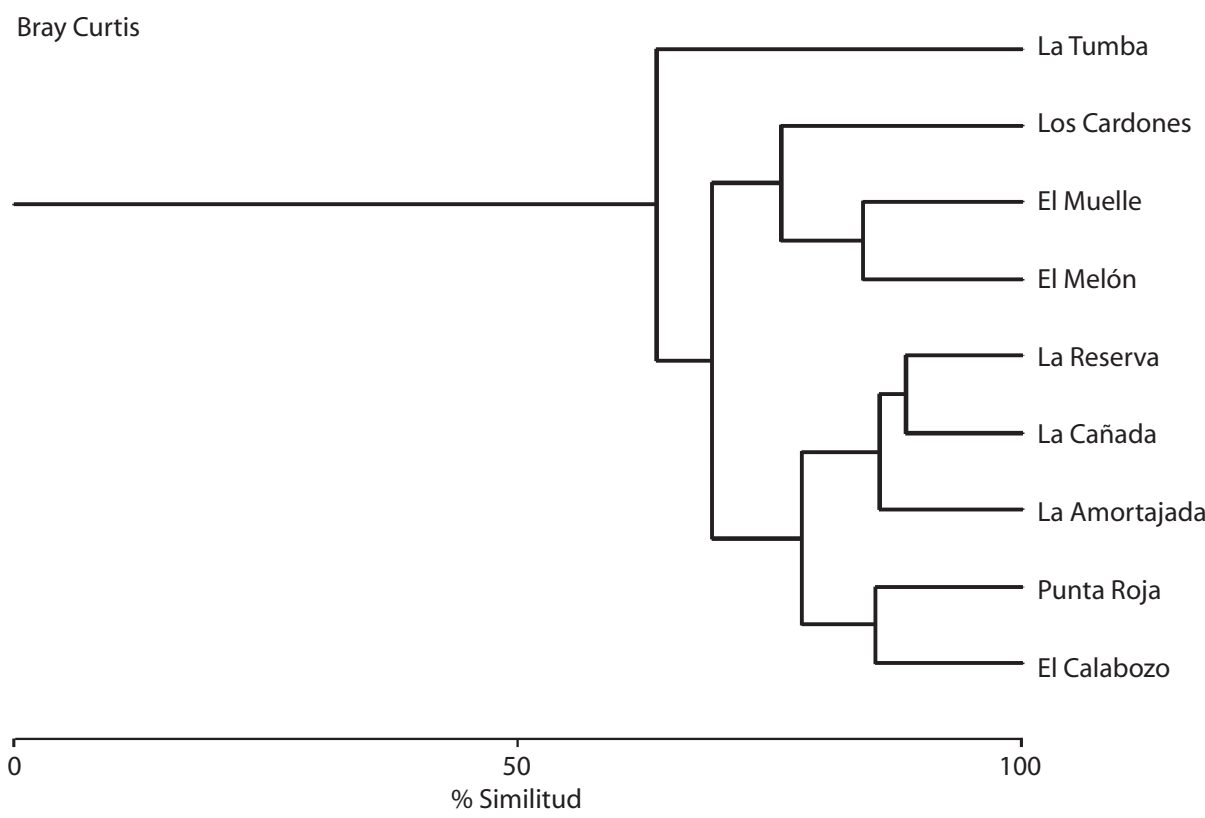

Fig. 4 Dendrograma del análisis de similitud entre las localidades de estudio. Se muestra la línea de corte (línea punteada).

alta diversidad. No obstante, se requieren más investigaciones, tanto en las aguas litorales someras, como en las aguas profundas para tener un mayor conocimiento de las especies que ahí habitan.

Los corales duros están mejor representados en la isla San José por Pocillopora verrucosa con $16 \%$ de abundancia relativa y en menor proporción por Porites panamensis con $5 \%$, caso contrario ocurre en la bahía de Loreto en que Porites predomina sobre Pocillopora. Ambas especies están ampliamente distribuidas en el litoral suroriental de la península de Baja California e islas aledañas (Squires 1959, Steinbeck y Ricketts 1971, Reyes-Bonilla 1990, Reyes-Bonilla y López-Pérez 1998).

Los moluscos estuvieron escasamente representados en los transectos, Pinna rugosa con el 2,2\%, Pinctada mazatlanica y Conus princeps con un $1,5 \%$ de abundancia relativa. Las tres especies son de interés económico. No obstante existen otras especies de importancia comercial en la isla San José observadas fuera de los transectos, como Crassostrea palmula, Hyotissa hyotis, Spondylus calcifer, Hexaplex brassica, Hexaplex princeps y Octopus bimaculatus.

\section{AGRADECIMIENTOS}

El presente estudio deriva de los apoyos recibidos del proyecto de investigación: "Estudio sobre la composición, estructura y función de las comunidades marinas litorales de la isla San José, B.C.S.", (CGPI-IPN, Clave: 990300) y gracias al apoyo económico procedente de los estímulos para el desempeño a la investigación (EDI) y COFAA del Instituto Politécnico Nacional.

\section{RESUMEN}

Se realizaron seis expediciones a la isla San José, Golfo de California entre los años 1999 y 2000 en donde se llevaron a cabo las observaciones y muestreos. Con base en los muestreos se determinó el componente específico de tres filos de macroinvertebrados bentónicos en nueve localidades: Cnidaria (Scleractinia y Gorgonacea), Mollusca (Gastropoda, Bivalvia y Cephalopoda), y Echinodermata (Echinoidea, Asteroidea y Holothuroidea). La abundancia del macrobentos conspicuo se estimó visualmente mediante buceo libre a una profundidad entre 2 y $6 \mathrm{~m}$ a lo largo de dos transectos en banda paralelos a la línea de costa. Se determinó un total de 38 táxones de los tres grupos. Las especies más abundantes son Tripneustes depressus (44,9\%), Echinometra vanbruntii (18,3), Phataria 
unifascialis (8,9\%), Centrostephanus coronatus $(8,0 \%)$, Diadema mexicanum (5\%) y Eucidaris thouarsii (3,6\%). No se encontraron diferencias significativas en la diversidad $\left(H^{\prime}\right)$, equidad $(J)$ y riqueza de especies $(S)$ entre los meses y localidades. El análisis de similitud identificó a dos grupos de las localidades del litoral oriental y occidental.

Palabras clave: Macroinvertebrados, Isla San José, Golfo de California.

\section{REFERENCIAS}

Baqueiro C.E. \& J.A. Massó. 1988. Variaciones poblacionales y reproducción de dos poblaciones de Chione undatella bajo diferentes regímenes de pesca en la Bahía de La Paz, BCS, México. Cienc. Pesq. INP. Sría de Pesca 6:51-67.

Baqueiro, C.E., I. Piña \& J.A. Massó. 1981. Análisis de una población sobreexplotada de Argopecten circularis en la Ensenada de La Paz, BCS, México. Cienc. Pesq. 2:57-65.

Brusca, R.C. 1980. Common invertebrates of the Gulf of California. 2nd Ed. Universidad de Arizona, Tucson, EEUU. 513 p.

Brusca, R.C. \& S. Trautwein. 2005. Cap. 4. Cnidaria and Ctenophora, p 49-65. In Hendrickx, Brusca y Findley (eds.). Listado y Distribución de la Macrofauna del Golfo de California, Mexico. Parte. 1. Invertebrados. Museo del Desierto Arizona-Sonora, Tucson, EEUU. 429 p.

Caso, M.E. 1979. Los equinodermos de la Bahía de Mazatlán, Sin. An. Centro Cienc. Mar y Limnol. Univ. Nal. Autón. México, México D.F., México. 6:197-368.

Caso, M.E. 1994. Estudio morfológico, taxonómico, ecológico y distribución geográfica de los asteroideos recolectados durante las campañas oceanográficas Cortés 1, 2, 3. An. Inst. Cienc. Mar y Limnol. Univ. Nal. Autón. México, México D.F., México. 12: 1-111.

Cintra-Buenrostro, C.E., H. Reyes-Bonilla \& O. Arizpe-Covarrubias. 1999. Los equinodermos (Echinodermata) del arrecife de Cabo Pulmo, Pacífico de México. Rev. Biol. Trop. 46: 341-344.

Conquest L., R. Burr, R. Donnelly, J. Chavarria \& V. Gallucci. 1996. Sampling methods for stock assessment for small-scale fisheries in developing countries 179-225. In: Gallucci et al. 1996 Stock assessment. Quantitative methods and applications for small scales fisheries. CRC, Lewis, Chelsea, Missouri, EEUU. 527 p.
Durham, J.W. \& J.L. Barnard. 1952. Stony corals of the eastern Pacific collected by Velero III and Velero IV. Allan Hancock Pac. Exped. 19:1-110.

Field, J. G., K. R. Clark y R. M. Warwick. 1982. A practical strategy for analysing multispecies distribution patterns. Mar. Ecol. Progr. Ser. 8:37-52.

García-Domínguez, F. 1991. Distribución, abundancia, reproducción y fauna asociada de la almeja roñosa Chione californiensis en la Ensenada de La Paz, BCS. Tesis de maestría CICIMAR-IPN, La Paz, BCS, México.

García-Domínguez, F. \& E.F. Félix-Pico. 1993. Gasterópodos depredadores de Chione californiensis (Broderip, 1835), en la Ensenada de La Paz, B.C.S., México. Rev. Universidad Michoacana, Morelia, México. 7: 28-31.

González-Medina, F.J., O.E. Holguin-Quiñones \& G. De la Cruz-Agüero. 2006. Variación espaciotemporal de algunos macroinvertebrados (Gastropoda, Bivalvia y Echinodermata) de fondos someros del archipiélago Espíritu Santo, Baja California Sur, México. Cienc Mar. 32: 33-44.

González-Navarro, E. \& J. Elorduy G. 1997. Bibliografía sobre la Bahía de La Paz, B.C.S., México, p 315-343 In Urbán, R. J. y M. Ramírez, R. (eds.). La Bahía de La Paz. Investigación y Conservación. UABCSCICIMAR-SCRIPPS, La Paz, BCS, México. 345 p.

Gotshall, D.W. 1987. Marine animals of Baja California. A guide to the common fishes and invertebrates. Sea Challengers, Monterey, California, EEUU. 112 p.

Hendrickx, M.E. \& R.C. Brusca. 2005. Cap. 15. Mollusca, p 195-310. In Hendrickx, Brusca y Findley (eds). Listado y Distribución de la Macrofauna del Golfo de California, Mexico. Parte. 1. Invertebrados. Museo Desierto Arizona-Sonora, Tucson, EEUU. 429 pp.

Holguin-Quiñones O.E., H. Wright \& F.A. Solís-Marín. 2000. Asteroidea, Echinoidea y Holothuroidea en fondos someros de la Bahía de Loreto, B.C.S., México. Rev. Biol. Trop. 48:749-757.

Holguin-Quiñones, O.E., E.F. Félix-Pico \& G. VillarealChávez. 2002. Crustáceos de franja litoral y fondos someros del Parque Marino Nacional Bahía de Loreto, BCS., p 157-182. In M.E. Hendrickx (ed.). Contribuciones al estudio de los crustáceos del Pacífico Este, vol.1. UNAM, México D.F, México.

Keen. A.M. 1971. Seashells of tropical west America. 2nd Ed. Stanford, California, EEUU. 1064 pp. 
Kerstitch A. \& H. Bertsch. 2007. Sea of Cortez Marine Invertebrates. A guide for the Pacific coast, México to Perú. $2^{\text {nd }}$ Ed. Sea Challengers. Monterey, California, EEUU. 124 pp.

Landa-Jaime, V., Michel-Morfín, J., Arciénega-Flores \& R. Flores-Vargas. 2005. Equinodermos del arrecife coralino de Tenacatita, Jalisco. Mem. XVIII Cong. Nal. Zool., Monterrey, Resumen 0237, Monterrey, México.

Magurran, A. E. 1988. Ecological diversity and its measurement. Princeton University Press, Nueva Jersey, EEUU.179 pp.

Maluf, L.Y. 1988. Composition and distribution of the Central Eastern Pacific echinoderms. Nat. Hist. Mus. Los Angeles. Tech. Rep. 2: 242 p.

Maluf, L.Y. \& R.C. Brusca. 2005. Cap. 18. Echinodermata, p 327-343. In Hendrickx, Brusca \& Findley (eds). Listado y Distribución de la Macrofauna del Golfo de California, Mexico. Parte. 1. Invertebrados. Museo Desierto Arizona-Sonora, Tucson, EEUU. 429 p.

Morris, P.A. 1966. A field guide to the Pacific coast shells. 2nd Ed. Houghton Mifflin. Boston, EEUU. 297 p.

Pielou, E. C. 1975. Ecological Diversity. Wiley Interscience, Nueva York, EEUU. 142 p.

Reyes-Bonilla, H. 1990. Distribución, riqueza específica, aspectos biogeográficos y taxonómicos de los corales hermatípicos del Golfo de California. Tes. Prof. Dpto. Biol. Mar., Univ. Aut. Baja Calif. Sur, California, EEUU. 128 p.

Reyes-Bonilla H. \& A. López-Pérez 1998. Biogeografía de los corales pétreos (Scleractinia) del Pacífico de México. Cienc Mar 24:211-224.

Reyes-Bonilla, H., L.E. Calderón, G. Cruz, P. Medina, R.A. López, M.D. Herrero, G.E. Leyte, A.L. Cupul \& J.D. Carriquiry. 2005. Atlas de Corales Pétreos (Anthozoa: Scleractinia) del Pacífico Mexicano. CICESE, CONABIO, CONACYT, UABCS, UdeG, UdelM, México.124 p.
Schwartzlose, R.A., D. Alvarez-Millán \& P. Brueggeman. 1992. Bibliografía de las Cienc Mar del Golfo de California. UABC, México. 425 p.

Solís-Marín, F.A., M.D. Herrero-Pérezrul, A. LaguardaFigueras \& J. Torres-Vega. 1993. Asteroideos y Equinoideos de México (Echinodermata), p 91-105. In S.I. Salazar-Vallejo \& N.E. González (eds.). Biodiversidad Marina y Costera de México. Com. Nal. Biodiversidad y CIQRO, México, 865 p.

Solís-Marín, F.A., H. Reyes-Bonilla, M.D. HerreroPérezrul, O. Arizpe-Covarrubias \& A. LaguardaFigueras. 1997. Sistemática y distribución de los equinodermos de la Bahía de La Paz. Cienc Mar. 23:249-263.

Solís-Marín, F.A., Laguarda-Figueras, A. de La Luz Durán-González, C. Ahearn Gust \& J. Torres Vega. 2005. Equinodermos (Echinodermata) del Golfo de California, México. Rev. Bio. Trop.53:123-137.

Squires, D.F. 1959. Corals and coral reefs in the Gulf of California. Bull. Am. Mus. Nat. Hist. Nueva York, EEUU. 118:370-431.

Steinbeck, J. \& E.F. Ricketts. 1971. Sea of Cortez. P. P. Appel, Mamoroneck, Nueva York, EEUU. 371$414 \mathrm{p}$.

Zamorano, P. \& G.E. Leyte-Morales. 2005. Cambios en la diversidad de equinodermos asociados al arrecife coralino de La Entrega, Oaxaca, México. Ciencia y Mar, 27:19-28.

\section{REFERENCIAS DE INTERNET}

McAleece, N. 1997. Biodiversity professional beta. The Natural History Museum \& The Scottish Association of Marine Science. biodiversity@nhm.ac.uk (consultado 20 abril 2008).

StatSoft. 2003. Statistica for windows, version 6.0. www. statsoft.com (consultado 20 abril 2008). 


\section{CLASE ASTEROIDEA}

Orden Valvatida Perrier, 1884

Familia Oreasteridae Fisher, 1911

Género Nidorellia Gray, 1840 Nidorellia armata (Gray, 1840)

Género Pentaceraster Döderlein, 1916

Pentaceraster cuminigi (Gray, 1840)

Familia Acanthasteridae Sladen, 1889

Género Acanthaster Gervais, 1841 Acanthaster planci (Linnaeus, 1758)

Familia Mithrodiidae Viguier, 1878

Género Mithrodia Gray, 1840 Mithrodia bradleyi (Verrill, 1870)

Familia Ophidiasteridae Verrill, 1870

Género Pharia Gray, 1840 Pharia pyramidatus (Gray, 1840)

Género Phataria Gray, 1840 Phataria unifascialis (Gray, 1840)

Orden Forcipulatida Perrier, 1884

Familia Heliasteridae Viguier, 1878

Género Heliaster Gray, 1840 Heliaster kubinijii (Xantus, 1860)

\section{CLASE ECHINOIDEA}

Orden Cidaroida Claus, 1880

Familia Cidariidae Gray, 1825

Género Eucidaris Pomel, 1883

Eucidaris thouarsii (Valenciennes, 1846)

Orden Diadematoida Duncan, 1889

Familia Diadematidae Gray, 1855

Género Diadema Gray, 1825

Diadema mexicanum (A. Agassiz, 1863)

Género Centrostephanus Peters, 1855

Centrostephanus coronatus (Verrill, 1867)

Orden Echinoida Claus, 1876

Familia Echinometridae Gray, 1825

Género Echinometra Gray, 1825

Echinometra vanbrunti (A. Agassiz, 1863)

Familia Toxopneustidae Troschel, 1872

Género Tripneustes L. Agassiz, 1841

Tripneustes depressus (A. Agassiz, 1863)

Género Toxopneustes A. Agassiz, 1841

Toxopneustes roseus (A. Agassiz, 1863) 
CLASE HOLOTHUROIDEA

Orden Aspidochirotida Grube, 1840

Familia Holothuriidae Ludwig, 1894

Género Holothuria Linnaeus, 1767

Subgénero Thymiosycia Pearson, 1914

Holothuria (Thymiosycia) arenicola (Semper, 1868)

Familia Stichopodidae Haeckel, 1896

Género Isostichopus Deichmann, 1958

Isostichopus fuscus (Ludwig, 1874)

\title{
SINOPSIS TAXONÓMICA DE LAS ESPECIES DE MOLUSCOS DE LA ISLA SAN JOSÉ, GOLFO DE CALIFORNIA
}

\author{
CLASE BIVALVIA
}

Orden Pterioida Newell, 1965

Familia Pteriidae Gray, 1857

Pinctada mazatlanica (Hanley, 1856)

Familia Pinnidae Leach, 1919

Pinna rugosa Sowerby, 1833

Orden Ostreoida de Férrusac, 1922

Familia Gryphaeidae Vayaloc, 1936

Hyotissa hyotis Linnaeus, 1758

Familia Ostreidae Rafinesque, 1915

Crassostrea palmula (Carpenter, 1857)

Myrakeena angelica (Rochebrune, 1895)

Familia Spondylidae Linnaeus, 1758

Spondylus calcifer Carpenter, 1857

Orden Venerida Adams, 1857

Familia Chamidae Blainville, 1825

Chama mexicana Carpenter, 1857

\section{CLASE GASTROPODA}

Orden Docoglossa Troschel, 1866

Familia Acmaeidae Carpenter, 1857

Collisella atrata (Carpenter, 1857)

Orden Neotaenioglossa Haller, 1882

Familia Cerithiidae de Férrusac, 1819

Cerithium maculosum Kiener, 1841

Familia Strombidae Rafinesque, 1815

Strombus galeatus Swainson, 1823

Familia Muricidae Rafinesque, 1815

Neorapana tuberculata (Sowerby, 1835)

Hexaplex princeps (Bropderip, 1822)

Hexaplex brasssica (Lamarck, 1822)

Familia Conidae Rafinesque, 1815

Conus princeps Linnaeus, 1758

Conus nux Broderip, 1833 


\section{CLASE CEPHALOPODA}

Familia Octopodidae Vaught, 1989

Octopus bimaculatus Verrill, 1883

\section{SINOPSIS TAXONÓMICA DE LAS ESPECIES DE CNIDARIOS DE LA ISLA SAN JOSÉ, GOLFO DE CALIFORNIA}

\section{CLASE ANTHOZOA}

Orden Scleractinia Bourne, 1900

Familia Pocilloporidae Gray, 1842

Pocillopora meandrina Dana, 1846

Pocillopora verrucosa (Ellis y Solander, 1786)

Familia Poritidae Gray, 1842

Porites panamensis Verrill, 1866

Familia Dendrophylliidae Gray, 1847

Tubastraea coccinea Lesson, 1829

Orden Gorgonacea

Familia Gorgoniidae

Muricea californica Aurivillius, 1931

Pacifigorgia agassizii (Verrill, 1864)

Gorgonia adamsi (Verrill, 1868) 
